\title{
Prognostic value of periostin in early-stage breast cancer treated with conserving surgery and radiotherapy
}

\author{
CHANGYOU LI ${ }^{1 *}$, JING XU $^{2 *}$, QI WANG $^{3}$, SHAOQING GENG $^{2}$, \\ ZHENG YAN $^{4}$, JIN YOU ${ }^{5},{\mathrm{ZHENFENG} \mathrm{LI}^{4} \text { and XIAO ZOU }}^{4}$ \\ ${ }^{1}$ Biotherapy Center; ${ }^{2}$ Department of Pathology, Qingdao Central Hospital; ${ }^{3}$ Department of Pathology, \\ Qingdao Cancer Hospital; ${ }^{4}$ Department of Breast Surgery; ${ }^{5}$ Anorectal Department, Qingdao Central Hospital, \\ The Second Affiliated Hospital of Qingdao University, Qingdao, Shandong 266042, P.R. China
}

Received April 5, 2016; Accepted December 8, 2017

DOI: $10.3892 / \mathrm{ol} .2018 .8310$

\begin{abstract}
The present study was performed to explore the prognostic significance of periostin expression in a cohort of patients with early-stage breast cancer treated with breast conserving surgery following radiotherapy. A tissue microarray of tumor samples from 259 patients with early-stage breast cancer was assayed for periostin, estrogen receptor (ER), progesterone receptor (PR), ErbB2 receptor tyrosine kinase 2 and Ki-67 expression by immunohistochemistry. The association of periostin with other clinicopathological parameters and clinical outcomes, including local recurrence free survival (RFS), distant metastasis free survival (DFS) and overall survival (OS), were assessed through log-rank tests and univariate and multivariate analysis. Periostin expression was identified in 91 of the 259 tissue samples (35\%). The periostin status was significantly associated with histological grade $(\mathrm{P}=0.001)$, nodal status $(\mathrm{P}=0.023)$, molecular subtype $(\mathrm{P}<0.01)$, ER status $(\mathrm{P}<0.01), \mathrm{PR}$ status $(\mathrm{P}<0.01)$ and $\mathrm{Ki}-67$ expression $(\mathrm{P}=0.011)$. Furthermore, periostin expression was associated with an increased risk of five-year local recurrence (95.8\% vs. $89.0 \% ; \mathrm{P}=0.017)$ and distant metastasis $(92.3 \%$ vs. 79.1\%; $\mathrm{P}=0.001$ ) in patients with early stage breast cancer. Multivariate analysis using Cox's proportional hazards model demonstrated that periostin expression was an independent predictor of all clinical outcomes in breast cancer (RFS, $\mathrm{P}=0.018$; DFS, $\mathrm{P}=0.025 ; \mathrm{OS}, \mathrm{P}=0.047)$. Therefore, it was concluded that periostin is associated with an increased risk of local relapse and distant metastasis in early-stage breast
\end{abstract}

Correspondence to: Dr Xiao Zou, Department of Breast Surgery, Qingdao Central Hospital, The Second Affiliated Hospital of Qingdao University, 127 Siliu South Road, Qingdao, Shandong 266042, P.R. China

E-mail: 1392365491@qq.com

${ }^{*}$ Contributed equally

Key words: periostin, breast cancer, radiotherapy, prognosis, conservative surgery cancer treated with conserving surgery and radiotherapy. This association should be further investigated in larger cohorts to validate the clinical significance of periostin expression.

\section{Introduction}

Breast cancer is the most common cause for cancer-associated mortality among women (1), and the treatment of breast cancer continues to present a difficult clinical challenge. Although radiotherapy with surgery may significantly prolong the survival period of patients with breast cancer, the 10-year loco-regional recurrence rate remains $38 \%$ for women $<40$ years; the reasons for this have yet to be established. The existence of loco-regional recurrence was associated with a worse distant disease-free survival and with a worse overall survival compared with those who did not (2). At present, the majority of patients with breast cancer in early stages are treated with breast conserving surgery followed by radiation therapy, with appropriate adjuvant systemic therapy. However, it remains a challenge for clinicians to predict the response to radiotherapy, and there is a demand to develop novel strategies to reduce tumor radioresistance. Therefore, an improved understanding of the molecular characteristics associated with intrinsic radioresistance is required. The tumor microenvironment has been increasingly recognized to serve an important role in the pathogenesis of breast cancer, in which stromal cells may produce extracellular matrix (ECM) components and growth factors to promote cancer cell activation, reprogramming and survival (3).

The ECM protein periostin (POSTN) is a bone adhesion molecule that regulates osteoblast adhesion and differentiation that may be upregulated in a wide range of cancer types, including colon, pancreatic, breast, gastric and non-small cell lung cancer, and neuroblastoma $(4,5)$. POSTN is required for cancer stem cell maintenance and may act as a critical regulator of tumorigenesis and progression $(6,7)$. It has been reported that the overexpression of POSTN is associated with metastasis and chemotherapy resistance by interacting with integrin receptors, as well as with other signals, particularly via the phosphoinositide 3-kinase/AKT pathway $(8,9)$.

A previous study demonstrated that POSTN protein expression was increased in $\mathrm{CD} 44^{+} / \mathrm{CD} 24^{-}$breast cancer stem cells 
compared with control cells, which was associated with cancer stem cell chemotherapy resistance (10). In the serum of patients with early-stage breast cancer, POSTN expression could be detected prior to surgery, and increased baseline serum POSTN levels were predictive of worse long-term survival outcomes in specific subgroups of patients (11). However, the relevance of POSTN expression status to clinical implications, particularly the response to radiotherapy for the management of breast cancer, is poorly understood.

In the present study, a tissue microarray (TMA) with tissue from 259 tumors from patients with breast cancer was used to evaluate the prognostic value of POSTN, including its association with clinical outcomes, and therefore, radiotherapy response. These data may provide clinical evidence of a method for predicting the tumor response to radiotherapy in patients with breast cancer and optimizing therapeutic strategies against radioresistance.

\section{Materials and methods}

Patient characteristics and histological review. Subsequent to obtaining approval from the institutional review board of Qingdao University School of Medicine Ethics Committee, 259 patients with early stage breast cancer were recruited to the study between January 2010 to January 2011 at the Department of Breast Surgery, Qingdao Central Hospital, the Second Affiliated Hospital of Qingdao University (Qingdao, China). All patients provided written informed consent prior to their inclusion in the study. Information about the patients' clinical history was obtained from the patient records database of Qingdao Central Hospital.

The age of each patient was defined at diagnosis. The size of the primary tumor was considered to be the largest tumor diameter reported by a pathologist subsequent to surgical excision. Patients with early stage breast cancer were staged as I and II based on the tumor-node-metastases (TNM) classification of the International Union against Cancer, revised in 2010 (12). The histological grade of the tumors was classified according to the World Health Organization criteria (13). The lymph node status was determined by the presence of histological evidence for lymph node metastasis. All patients in the present study were treated with breast conserving surgery with or without axillary lymph node dissection. Following surgery, the patients received standard whole breast irradiation at the radiation oncology facilities of the Department of Therapeutic Radiology, Qingdao Central Hospital, the Second Affiliated Hospital of Qingdao University. The median total dose was 50 Gy to the whole breast. Adjuvant systemic chemotherapy and/or adjuvant hormone therapy were administered as clinically indicated in accordance with standard practice during the time of the study. Local recurrence-free survival (RFS), distant metastasis-free survival (DFS) and overall survival (OS) were the endpoints evaluated.

TMA construction. Formalin-fixed, paraffin-embedded tissues from the Department of Pathology, Qingdao Central Hospital were used in the construction of the breast cancer sample TMA. Briefly, representative areas of breast tumor samples were selected from whole tissue sections by pathologists. A cylindrical specimen core of 2-mm diameter was extracted from these regions and precisely arrayed into a new recipient paraffin block using a custom-built precision instrument (Beecher Instruments; Estigen OÜ, Tartu, Estonia) as previously described (14). The resulting TMA blocks were cut to 4- $\mu \mathrm{m}$ thickness with a microtome and placed on slides with an adhesive tape-transfer method (InstruMedics; Stryker Corporation, Kalamazoo, MI, USA). One section from each TMA was stained with hematoxylin and eosin (in hematoxylin for up to $2 \mathrm{~min}$ and eosin for $30 \mathrm{sec}$ at room temperature) to verify the presence of tumor cells. The TMA was assessed for the staining intensity, and the percentage of stained cells in the nucleus, cytoplasm or membrane.

Immunohistochemical study. Immunohistochemical analysis was performed on the $4-\mu \mathrm{m}$ thick paraffin-embedded tissue sections of the TMA using a standard streptavidin-peroxidase method. The subsequent levels of tissue sections were stained with Ventana Benchmark XT Autostainer according to the manufacturer's instructions (Ventana Medical Systems, Inc., Tucson, AZ, USA). The slides were incubated with a rabbit anti-human primary antibody against POSTN (dilution 1:500, cat. no. sc-67233; Santa Cruz Biotechnology, Inc., Dallas, TX, USA), ER (cat. no. G07268), PR (cat. no. Y12992), HER2 (cat. no. Y08422) and Ki-67 (cat. no. Y13569) (all from Roche Diagnostics, Indianapolis, IN, USA) for 28-32 min at $37^{\circ} \mathrm{C}$. The primary antibodies for ER, PR, HER2, Ki-67 and the goat anti-rabbit horseradish peroxidase-conjugated secondary antibody (Ultraview Universal DAB detection kit; cat. no. 05269806001; Roche Diagnostics) were ready-to-use for $8 \mathrm{~min}$ at $37^{\circ} \mathrm{C}$. A known positive case was stained as a positive control. The primary antibody was replaced with nonimmune mouse serum (Santa Cruz Biotechnology, Inc.) as a negative control. Immunohistochemical staining was developed in a Dako Autostainer Plus using an LSAB detection kit (Dako; Agilent Technologies, Inc., Santa Clara, CA, USA). The individuals performing immunohistochemical evaluation were blinded to the clinical information. The staining results for all markers were first determined independently, then reviewed together at a multi-head microscope. According to the criteria for immunohistochemical evaluation reported previously (10), the presence of $1 \%$ of positively stained neoplastic cells was defined as positive POSTN expression.

Statistical analysis. Statistical analysis was performed using SPSS 22.0 (IBM Corp., Armonk, NY, USA). The association of POSTN expression with categorical clinicopathological parameters was assessed by $\chi^{2}$ tests. Estimates of RFS, DFS and OS were calculated by the Kaplan-Meier product-limit method, and the differences were assessed by the log-rank test. Multivariate analysis was performed using the Cox proportional hazards regression model to determine the prognostic effect on the independent contribution of each variable to survival; hazard ratios (HRs) and their corresponding 95\% confidence intervals (CIs) were calculated. All $\mathrm{P}$-values are two-sided; $\mathrm{P}<0.05$ was considered to indicate a statistically significant difference.

\section{Results}

Immunostaining results of POSTN in breast cancer tissues. The intracellular localization of POSTN protein staining 


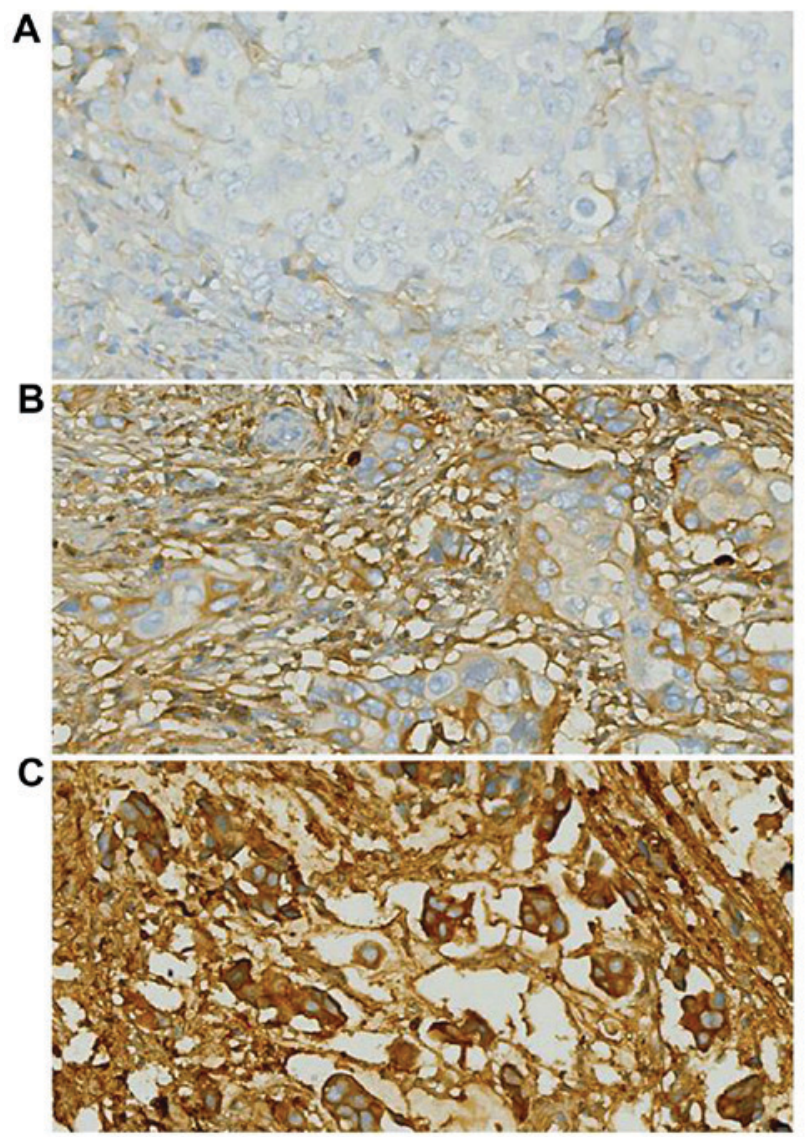

Figure 1. Representative images of immunohistochemical staining of POSTN in breast cancer tissues, including (A) negative, (B) moderate and (C) high expression of POSTN. Magnification, x400. POSTN, periostin.

was examined, as its location may affect the mechanism of action and potentially, the response to treatment. In the breast cancer tissue samples, there was a heterogeneous distribution of POSTN in the cytoplasm. Nuclear POSTN expression was relatively infrequent. Representative POSTN immunostaining results are included in Fig. 1.

Descriptive statistics. The clinical and pathological variables of the patients are included in Table I. The median age at diagnosis for all patients was 53 years (range, 27-93), with $44.4 \%$ of the patients $(n=115)<50$ years at the time of diagnosis. A total of $45 \%(n=117)$ of the patients presented with lymph node metastases (N1 or 2) at the time of surgery. Of the 259 patients, $27 \%$ had the Luminal A molecular subtype, 33\% Luminal B, 13\% HER2-overexpressed and 25\% triple-negative; the remaining patients were uncategorized.

As of February 2015, the median follow-up time was 51 months. Of all the patients in the study, $7 \%(n=17)$ of patients experienced local relapse, $12 \%(n=32)$ experienced distant metastasis and $11 \%(\mathrm{n}=29)$ succumbed to the disease.

POSTN expression in breast cancer and its association with clinicopathological characteristics. Clinicopathological characteristics and molecular features were compared with the POSTN expression status for the patients in the cohort of the present study. The results are summarized in Table I. The POSTN status was significantly associated with the

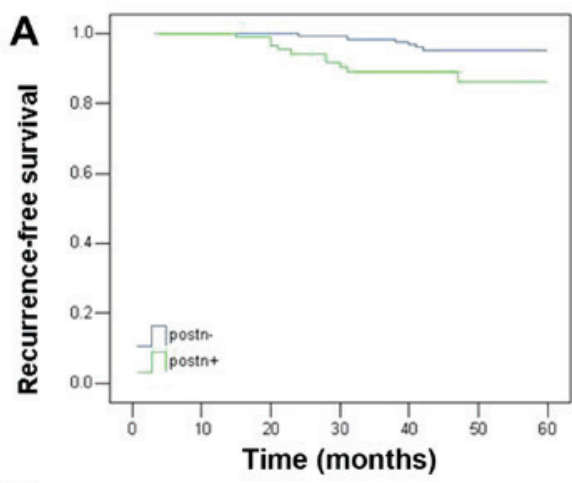

B
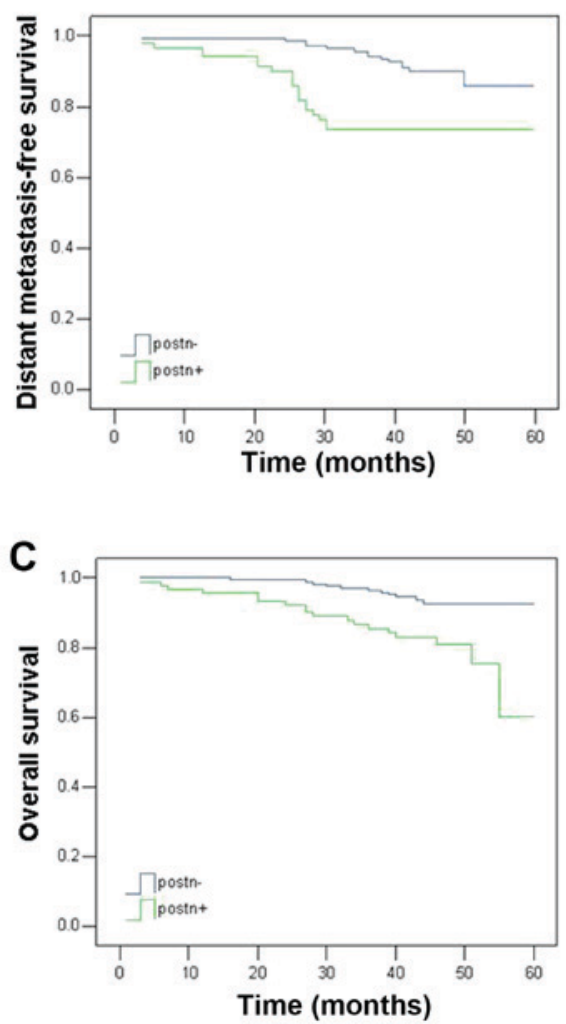

Figure 2. Kaplan-Meier survival curves of five-year clinical outcomes stratified by POSTN expression in patients with breast cancer, including (A) local recurrence free survival, (B) distant metastasis free survival and (C) overall survival. POSTN, periostin.

histological grade $(\mathrm{P}<0.01)$, nodal status $(\mathrm{P}<0.05)$, molecular subtype $(\mathrm{P}<0.01)$, estrogen receptor $(\mathrm{ER})$ status $(\mathrm{P}<0.01)$, progesterone receptor $(\mathrm{PR})$ status $(\mathrm{P}<0.01)$ and $\mathrm{Ki}-67$ expression $(\mathrm{P}<0.05)$. No significant association between POSTN expression and other parameters could be established.

Association between POSTN and postoperative local relapse and distant metastasis. Five-year survival analysis was performed for the 259 patients, considering the RFS, DFS and OS rates. As summarized in Table II and illustrated in Fig. 2, tumors with positive POSTN expression were associated with poorer outcomes for all endpoints, including RFS $(\mathrm{P}<0.05)$, DFS $(\mathrm{P}<0.01)$ and OS $(\mathrm{P}<0.01)$.

Prognostic analysis of POSTN expression in breast cancer. Multivariate analysis with the Cox proportional hazards 
Table I. Clinicopathological features of 259 patients with breast cancer, and the association with the expression of POSTN.

\begin{tabular}{|c|c|c|c|c|}
\hline \multirow[b]{2}{*}{ Clinicopathological feature } & \multirow[b]{2}{*}{$\mathrm{n}(\%)$} & \multicolumn{2}{|c|}{ POSTN expression, $\mathrm{n}$} & \multirow[b]{2}{*}{ P-value } \\
\hline & & Negative & Positive & \\
\hline Total & 259 & 168 & 91 & \\
\hline Age (years) & & & & 0.248 \\
\hline$\leq 50$ & $115(44.4)$ & 79 & 36 & \\
\hline$>50$ & $144(55.6)$ & 89 & 55 & \\
\hline Tumor size & & & & 0.123 \\
\hline $\mathrm{T} 1$ & $142(54.8)$ & 98 & 44 & \\
\hline $\mathrm{T} 2$ & $117(45.2)$ & 70 & 47 & \\
\hline Histological grade & & & & $0.001^{\mathrm{a}}$ \\
\hline I & $29(11.2)$ & 26 & 3 & \\
\hline II & $160(61.8)$ & 106 & 54 & \\
\hline III & $70(27.0)$ & 36 & 34 & \\
\hline Nodal status & & & & $0.023^{\mathrm{a}}$ \\
\hline Negative & $146(56.4)$ & 99 & 47 & \\
\hline Positive & $113(43.6)$ & 61 & 52 & \\
\hline Molecular subtype & & & & $<0.001^{\mathrm{a}}$ \\
\hline Luminal A & $71(27.4)$ & 59 & 12 & \\
\hline Luminal B & $84(32.4)$ & 55 & 29 & \\
\hline HER2-overexpressed & $34(13.1)$ & 22 & 12 & \\
\hline Triple-negative & $65(25.1)$ & 28 & 37 & \\
\hline Unclassified & $5(1.9)$ & 4 & 1 & \\
\hline Estrogen receptor status & & & & $<0.001^{\mathrm{a}}$ \\
\hline Negative & $106(40.9)$ & 54 & 52 & \\
\hline Positive & $153(59.1)$ & 114 & 39 & \\
\hline Progesterone receptor status & & & & $<0.001^{\mathrm{a}}$ \\
\hline Negative & $124(47.9)$ & 67 & 57 & \\
\hline Positive & $135(52.1)$ & 101 & 34 & \\
\hline HER2 status & & & & 0.897 \\
\hline Negative & $178(68.7)$ & 115 & 63 & \\
\hline Positive & $81(31.3)$ & 53 & 28 & \\
\hline Ki-67 expression & & & & $0.011^{\mathrm{a}}$ \\
\hline Low & $104(40.2)$ & 77 & 27 & \\
\hline High & $155(59.8)$ & 91 & 64 & \\
\hline Local recurrence & & & & $0.034^{\mathrm{a}}$ \\
\hline No & $242(93.4)$ & 161 & 81 & \\
\hline Yes & $17(6.6)$ & 7 & 10 & \\
\hline Postoperative distant metastasis & & & & $0.002^{\mathrm{a}}$ \\
\hline Negative & $227(87.6)$ & 155 & 72 & \\
\hline Positive & $32(12.4)$ & 13 & 19 & \\
\hline Survival status & & & & $0.001^{\mathrm{a}}$ \\
\hline Alive & $230(88.8)$ & 157 & 73 & \\
\hline Deceased & $29(11.2)$ & 11 & 18 & \\
\hline
\end{tabular}

${ }^{\mathrm{a}} \mathrm{P}<0.05$. POSTN, periostin; HER2, ErbB2 receptor tyrosine kinase 2.

model was performed using the following variables: POSTN expression, age, tumor size, histological grade, nodal status, disease subtype, ER, PR and HER2 status, and Ki-67 expression (Table III). Positive POSTN expression was associated with a relatively poor outcome for all endpoints. A larger tumor size, the tumor subtype and high $\mathrm{Ki}-67$ expression were 
Table II. Five-year outcomes as a function of POSTN expression.

Patients with POSTN status $(\%)$

Clinical outcome

Negative Positive P-value

\begin{tabular}{llll}
\hline Local recurrence-free survival & 95.8 & 89.0 & 0.017 \\
Distant metastasis-free survival & 92.3 & 79.1 & 0.001 \\
Overall survival & 93.5 & 80.2 & 0.001 \\
\hline
\end{tabular}

POSTN, periostin.

associated with reduced RFS time $(\mathrm{P}=0.007, \mathrm{P}=0.005$ and $\mathrm{P}=0.012$, respectively). Positive nodal status was also associated with a reduced DFS and $\mathrm{OS}$ time $(\mathrm{P}=0.005$ and $\mathrm{P}=0.003$, respectively). A higher histological grade and high $\mathrm{Ki}-67$ expression were further associated with a reduced OS time $(\mathrm{P}=0.043$ and $\mathrm{P}=0.016$, respectively).

Therefore, it was demonstrated by the multivariate analysis that POSTN expression retained significance independent of other prognostic factors, including as an independent prognostic marker for local relapse, distant metastasis and OS in the patients with early stage breast cancer of the present study.

\section{Discussion}

Radiation therapy subsequent to conserving surgery has made notable contributions to oncotherapy; it remains the standard therapeutic modality for breast cancer patients in early stages, and has been demonstrated to improve the overall survival (15). Nevertheless, tumor relapse and therapy failure continue to occur in a high proportion of patients. One emerging explanation posits that cancer stem cells may be responsible for the resistance to chemotherapeutic agents and radiation therapy (16). The exposure to radiation may reprogram differentiated breast cancer cells into induced breast cancer stem cells, which express the same stemness-related genes and exhibit enhanced tumorigenicity compared with non-irradiated samples (17). Phillips et al (18) previously reported that $\mathrm{CD} 44^{+} \mathrm{CD} 24^{- \text {/low }}$ breast cancer stem cells are less radiosensitive, supporting the hypothesis that cancer stem cells are more radioresistant than non-stem cancer cells. Specifically, breast cancer stem cells with the HER $2^{+} / \mathrm{CD} 44^{+} / \mathrm{CD} 24^{-/ \mathrm{low}}$ phenotype exhibited increased aggressiveness, tumorigenesis and radioresistance compared with HER $2{ }^{-} / \mathrm{CD} 44^{+} / \mathrm{CD} 24^{-/ \text {low }}$ breast cancer cells, thus providing a potential therapeutic target for the radiosensitization of breast cancer cells (19). One mechanism for radioresistance in cancer stem cells appears to be associated with their enhanced DNA repair capacity, reactive oxygen species defenses and self-renewal potential (20).

The extracellular matrix protein POSTN is highly expressed in a subset of basal-like breast cancer (BLBC) cell lines, as well as in cancer stem cell-enriched populations within tumors. When produced by cancer stem cells, POSTN acts to maintain the stem-like state through the activation of integrin receptors and the production of the key cytokines,

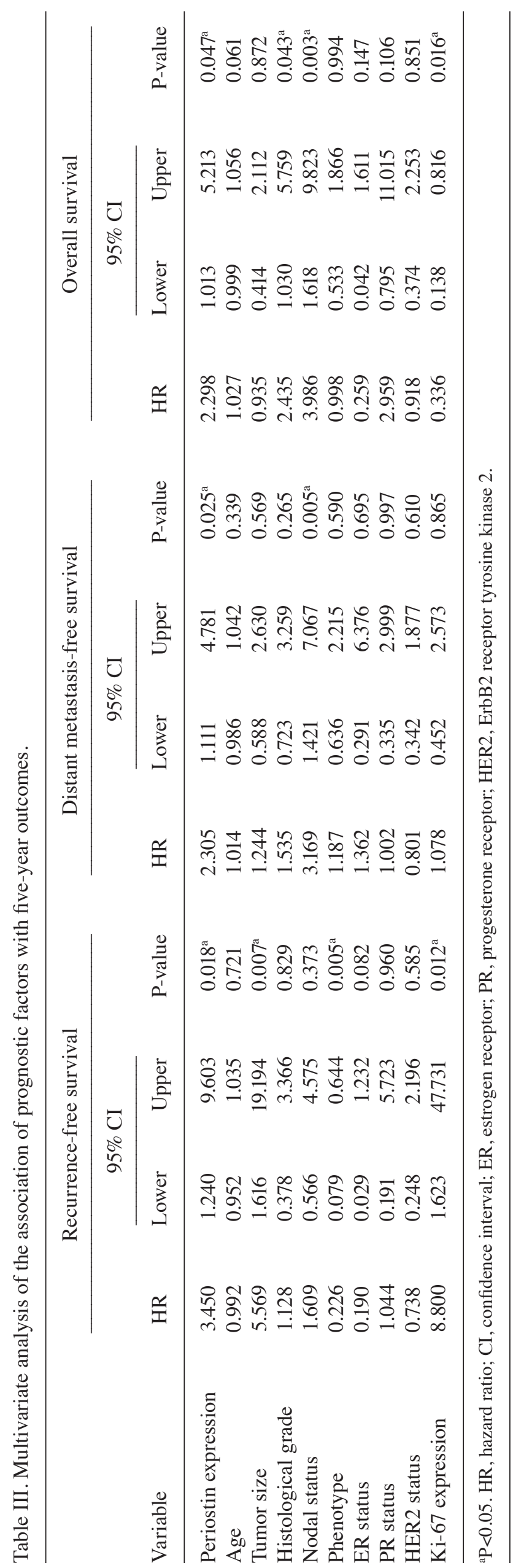


interleukin-6 and -8 (21). POSTN is required for cancer stem cell maintenance and increases Wnt signalling in cancer stem cells, and therefore, is a critical limiting factor during metastatic colonization (6).

Recent studies have demonstrated that POSTN may be critical for the interactions between cancer stem cells and their metastatic niche (4). It is necessary for cancer stem cells to maintain vitality, and blocking its function prevents metastasis (7). Xu et al (10) reported that POSTN was highly expressed in cancer stem cells and could be a potential biomarker for the bone metastasis and chemotherapy resistance of breast cancer tumors. To the best of our knowledge, no study has examined the relationship between POSTN, radioresistance and the prognosis of patients with breast cancer receiving radiotherapy.

In the present study, in a cohort of breast cancer patients treated with conserving surgery and radiation therapy, positive POSTN expression was associated with higher rates of local recurrence and distant metastasis. It was considered to be an independent predictor for all endpoints based on multivariate analysis, and therefore, may be suitable as a biomarker of radioresistance. An association was also identified between POSTN expression and certain clinicopathological characteristics of the patients with breast cancer, although previous studies have reported that serum POSTN levels are not associated with clinicopathological parameters in early-stage colorectal cancer, lung cancer and hepatocellular carcinoma (22-24). However, Sasaki et al (25) reported on the presence of elevated levels of serum POSTN in patients with breast cancer affected by bone metastases, suggesting that serum POSTN may represent a marker of bone metastasis in patients with breast cancer. The present study identified that POSTN expression in the tumor was associated with histological grade, nodal status, molecular subtype, ER status, PR status and Ki-67 levels in these patients. In addition, through survival analysis, positive POSTN expression was associated with a higher propensity for local and distant relapse and poorer overall survival. Cox's proportional hazard regression model analysis identified POSTN as an independent prognostic factor for breast cancer outcomes following conserving surgery and radiotherapy. This suggests that POSTN may be critical for breast cancer tumorigenesis, and may be a potential biomarker for the metastasis and radiotherapy resistance of breast cancer.

The limitations of the present study include a relatively small sample size and a short follow-up time. Further studies with a larger number of subjects will be required. In addition, a more detailed analysis with a focus on identifying associations between POSTN expression and cohort subgroups, particularly patients with triple-negative breast cancer treated with radiotherapy, will be required. The association of POSTN expression with the breast cancer stem cell ratio in tissues, and the molecular mechanisms by which POSTN regulates the effect of cancer stem cells in the radiotherapeutic resistance of breast tumors, also require more extensive investigation.

\section{Acknowledgements}

The present study was supported by Qingdao Health Science and Technology Project (grant no. 2016-WJZD039).

\section{Competing interests}

The authors declare that they have no competing interests.

\section{References}

1. Gaffan J, Dacre J and Jones A: Educating undergraduate medical students about oncology: A literature review. J Clin Oncol 24:1932-1939, 2006.

2. Bollet MA, Sigal-Zafrani B, Mazeau V, Savignoni A, de la Rochefordière A, Vincent-Salomon A, Salmon R, Campana F, Kirova YM, Dendale R and Fourquet A: Age remains the first prognostic factor for loco-regional breast cancer recurrence in young $(<40$ years) women treated with breast conserving surgery first. Radiother Oncol 82: 272-280, 2007.

3. Hanahan D and Coussens LM: Accessories to the crime: Functions of cells recruited to the tumor microenvironment. Cancer Cell 21: 309-322, 2012.

4. Wang Z and Ouyang G: Periostin: A bridge between cancer stem cells and their metastatic niche. Cell Stem Cell 10: 111-112, 2012.

5. Zhang Y, Zhang G, Li J, Tao Q and Tang W: The expression analysis of periostin in human breast cancer. J Surg Res 160: 102-106, 2010

6. Malanchi I, Santamaria-Martínez A, Susanto E, Peng H, Lehr HA, Delaloye JF and Huelsken J: Interactions between cancer stem cells and their niche govern metastatic colonization. Nature 481: 85-89, 2011

7. Kyutoku M, Taniyama Y, Katsuragi N, Shimizu H, Kunugiza Y, Iekushi K, Koibuchi N, Sanada F, Oshita Y and Morishita R: Role of periostin in cancer progression and metastasis: Inhibition of breast cancer progression and metastasis by anti-periostin antibody in a murine model. Int J Mol Med 28: 181-186, 2011.

8. Xiao ZM, Wang XY and Wang AM: Periostin induces chemoresistance in colon cancer cells through activation of the pi3k/akt/survivin pathway. Biotechnol Appl Biochem 62: 401-406, 2015.

9. Sung PL, Jan YH, Lin SC, Huang CC, Lin H, Wen KC, Chao KC, Lai CR, Wang PH, Chuang CM, et al: Periostin in tumor microenvironment is associated with poor prognosis and platinum resistance in epithelial ovarian carcinoma. Oncotarget 7: 4036-4047, 2016.

10. Xu D, Xu H, Ren Y, Liu C, Wang X, Zhang H and Lu P: Cancer stem cell-related gene periostin: A novel prognostic marker for breast cancer. PLoS One 7: e46670, 2012.

11. Nuzzo PV, Rubagotti A, Argellati F, Di Meglio A, Zanardi E, Zinoli L, Comite P, Mussap M and Boccardo F: Prognostic value of preoperative serum levels of periostin (PN) in early breast cancer (BCa). Int J Mol Sci 16: 17181-17192, 2015.

12. American Joint Committee on Cancer: Cancer Staging Handbook: From the AJCC Cancer Staging Manual. Edge SB, Byrd DR, Compton CC, Fritz AG, Greene F and Trotti A (eds). 7th edition. Springer-Verlag, New York, pp347, 2010.

13. Lakhani SR, Ellis IO, Schnitee SJ, Tan PH and van de Vijver MJ (eds): WHO Classification of Tumours of the Breast. Vol 4. 4th edition. IARC Press, Lyon, 2012.

14. Diallo-Danebrock R, Ting E, Gluz O, Herr A, Mohrmann S, Geddert H, Rody A, Schaefer KL, Baldus SE, Hartmann A, et al: Protein expression profiling in high-risk breast cancer patients treated with high-dose or conventional dose-dense chemotherapy. Clin Cancer Res 13: 488-497, 2007.

15. Gebski V, Lagleva M, Keech A, Simes J and Langlands AO: Survival effects of postmastectomy adjuvant radiation therapy using biologically equivalent doses: A clinical perspective. J Natl Cancer Inst 98: 26-38, 2006.

16. Rosen JM and Jordan CT: The increasing complexity of the cancer stem cell paradigm. Science 324: 1670-1673, 2009.

17. Lagadec C, Vlashi E, Della Donna L, Dekmezian C and Pajonk F: Radiation-induced reprogramming of breast cancer cells. Stem Cells 30: 833-844, 2012.

18. Phillips TM, McBride WH and Pajonk F: The response of $\mathrm{cd} 24^{- \text {low }} / \mathrm{cd} 44^{+}$breast cancer-initiating cells to radiation. J Natl Cancer Inst 98: 1777-1785, 2006.

19. Duru N, Fan M, Candas D, Menaa C, Liu HC, Nantajit D, Wen Y, Xiao K, Eldridge A, Chromy BA, et al: HER2-associated radioresistance of breast cancer stem cells isolated from HER2-negative breast cancer cells. Clin Cancer Res 18: 6634-6647, 2012.

20. Rycaj K and Tang DG: Cancer stem cells and radioresistance. Int J Radiat Biol 90: 615-621, 2014. 
21. Lambert AW, Wong CK, Ozturk S, Papageorgis P, Raghunathan R, Alekseyev Y, Gower AC, Reinhard BM, Abdolmaleky HM and Thiagalingam S: Tumor cell-derived periostin regulates cytokines that maintain breast cancer stem cells. Mol Cancer Res 14: 103-113, 2016.

22. Ben QW, Zhao Z, Ge SF, Zhou J, Yuan F and Yuan YZ: Circulating levels of periostin may help identify patients with more aggressive colorectal cancer. Int J Oncol 34: 821-828, 2009.

23. Hong L, Sun H, Lv X, Yang D, Zhang J and Shi Y: Expression of periostin in the serum of nsclc and its function on proliferation and migration of human lung adenocarcinoma cell line (a549) in vitro. Mol Biol Rep 37: 2285-2293, 2010.
24. Lv Y, Wang W, Jia WD, Sun QK, Huang M, Zhou HC, Xia HH, Liu WB, Chen H, Sun SN and Xu GL: High preoparative levels of serum periostin are associated with poor prognosis in patients with hepatocellular carcinoma after hepatectomy. Eur J Surg Oncol 39: 1129-1135, 2013.

25. Sasaki H, Yu CY, Dai M, Tam C, Loda M, Auclair D, Chen LB and Elias A: Elevated serum periostin levels in patients with bone metastases from breast but not lung cancer. Breast Cancer Res Treat 77: 245-252, 2003. 